\title{
Gene Expression Profiles of Programmed Death-1 (+) Lymphocytes in Peripheral Blood Reveal their Significance in Tumor Immunity of T Cell Non-Hodgkin Lymphoma
}

\author{
Zhang $\mathbf{W}^{1 \#}$, Zuo $\mathbf{M}^{1,2 \#}$, Shen $\mathrm{H}^{1}$, Yin $\mathrm{J}^{1}$, Wang $\mathbf{W}^{1}$, Zhang $\mathrm{Y}^{1}$ and Zhou $\mathrm{D}^{1 *}$ \\ ${ }^{1}$ Department of Hematology, Peking Union Medical College Hospital, Beijing, PR China \\ ${ }^{2}$ Department of Minimally invasive intervention, Sun Yat-Sen University Cancer Center, Guangzhou, China
}

\#The authors contributed equally to this work

\begin{abstract}
Purpose: The aim of this study was to analyze the gene expression profiles of peripheral blood PD-1 (+) lymphocytes in T cell non-Hodgkin lymphoma (T-NHL) and explored its potential clinical significance.

Experimental designs: 10 patients newly diagnosed with T-NHL and 10 healthy controls were enrolled. The peripheral blood PD-1 (+) lymphocytes was sorted by magnetic beads, and then RNA was extracted for digital gene expression profile (DGE)

Results: Compared with healthy controls, there were 5195 genes differently expressed in PD-1 (+) lymphocytes in peripheral blood of T-NHL patients, among which 3321 genes were highly expressed and 1874 genes were lowly expressed. Enrichment analysis showed that the function of differently expressed genes was related to cell proliferation/apoptosis, immune response, metabolism and cell adhesion. And the signaling pathway mainly involved cell cycle, TCR and TLR signaling pathway.
\end{abstract}

Conclusion: The gene expression profile of PD-1 (+) lymphocytes in peripheral blood of newly diagnosed T-NHL patients was obviously specific, indicating that these lymphocytes might play important roles in tumor immunity.

Keywords: T cell non-Hodgkin lymphoma; Programmed death-1; Peripheral blood lymphocytes; Tumor immunity

\section{Introduction}

T cell non-Hodgkin's lymphoma is an aggressive subtype of nonHodgkin's lymphoma (NHL), which is of high degree malignancy. The patients who received first-line chemotherapy are prone to be relapsed, and once happened, they lack of effective salvage therapy. So the 5-year overall survival rate is only $20-40 \%$ [1-3]. However, the incidence of T-NHL in China is significantly higher than that in the west countries, accounting for $15-20 \%$ of all malignant lymphomas [1]. Therefore, it is of great clinical significance to explore the pathogenesis and therapy of T-NHL in China.

In recent years, tumor immunity has become a hot spot in lymphoma research. A variety of studies [4] show that the function of immune cells in tumor microenvironment are defect, which may be related to tumor immune tolerance. Programmed death-1 (PD1), a member of $\mathrm{T}$ cell receptor CD28 family, is a most valuable and promising molecule in the study of tumor immune escape [5]. Once stimulated by antigens, PD- 1 is widely expressed in immune cells, such as T lymphocytes, B lymphocytes and NK cells, and then act as the "immune brake", which can avoid autoimmune phenomenon caused by excessive activation of immune cells [6]. But in tumor microenvironment, PD-1 will excessively inhibit immune system, contributing to tumor immune escape [7]. Actually these findings have brought a new breakthrough in tumor therapy. PD-1 inhibitors have showed great success in melanoma, kidney cancer, lung cancer and other solid tumors, and improved the prognosis of patients to a large extent. Besides, PD-1 inhibitor gradually begins to be applied to the treatment of Hodgkin's lymphoma, follicular lymphoma and refractory recurrent B-cell lymphoma [8-10].

However, studies about PD-1 in T-cell lymphoma are still in the primary stage. As we all know that $\mathrm{T}$ cell lymphoma display a very strong heterogeneity, so the tumor microenvironment might also differ among subtypes. Besides, in tumor microenvironment of T-NHL, PD-1 is not only expressed on lymphocytes, but also expressed in some tumor cells, making the condition become more complex. Recent years, researchers begin to explore the function of PD-1 in a relatively stable environment, the peripheral blood. A number of studies have shown that there is a significant difference in the expression of PD-1 on peripheral blood lymphocytes between lymphoma patients, which is associated with disease prognosis and treatment response [11-13]. On the basis of this, we utilized digital gene expression profiling to analyze gene expression profile of peripheral blood PD-1 (+) lymphocyte in T-NHL patients, and tried to figure out what kind of biological process PD-1 (+) lymphocytes were correlated with, and whether they were involved in tumor immunity.

\section{Patients and Methods}

\section{Patients}

Our study included 10 newly diagnosed T-NHL patients in Peking Union Medical College Hospital (PUMCH) from December

*Corresponding author: Zhou D, Department of Hematology, Peking Union Medical College Hospital, Chinese Academy of Medical Sciences and Peking Union Medical College, No. 1 Shuaifuyuan, Beijing 100730, PR China, Tel: 01069155024; E-mail: zhoudbpumc@126.com

Received March 16, 2018; Accepted August 31, 2018; Published September 05 2018

Citation: Zhang W, Zuo M, Shen H, Yin J, Wang W, et al. (2018) Gene Expression Profiles of Programmed Death-1 (+) Lymphocytes in Peripheral Blood Reveal thei Significance in Tumor Immunity of T Cell Non-Hodgkin Lymphoma. J Blood Lymph 8: 223. doi:10.4172/2165-7831.1000223

Copyright: @ 2018 Zhang W, et al. This is an open-access article distributed unde the terms of the Creative Commons Attribution License, which permits unrestricted use, distribution, and reproduction in any medium, provided the original author and source are credited. 
2016 to May 2017. The patients were confirmed with the 2008 WHO classification criteria [14] and excluded based on cytomegalovirus (CMV), Epstein-Barr virus (EBV) infection and autoimmunity systematic disease. In addition, 10 healthy people who were randomly selected from the PUMCH Health Examination Center were included as the control group. This study had been approved by the Ethics Committee of PUMCH and both patients and healthy controls had signed informed consent.

\section{Magnetic beads sorting the PD-1 (+) lymphocytes}

$20 \mathrm{ml}$ of EDTA anticoagulated peripheral blood was collected from patients and healthy controls, and treated within 2 hours. About $10^{7} \sim 10^{8}$ mononuclear cells were collected and incubated with PD-1$\mathrm{PE}$ antibody at $4^{\circ} \mathrm{C}$ for $20 \mathrm{~min}$ in the dark, washed twice with $2 \mathrm{~mL}$ PBS cells ( $300 \mathrm{~g}, 5 \mathrm{~min}$ ), and then incubated with anti-PE microbeads at $4^{\circ} \mathrm{C}$ for $20 \mathrm{~min}$. Finally the PD-1 (+) lymphocytes were obtained by magnetic separation, and dissolved with Trizol for storage. PD1-PE antibody (clone: PD1.3.1.3; PE) and anti-PE microbeads were purchased from Miltenyi Biotec, Germany.

\section{RNA extraction and detection}

Total RNA was extracted from dissolving solution by RNA Kit (Qiagen GmbH, Hilden, Germany). RNA degradation and contamination was monitored on $1 \%$ agarose gels, RNA purity was checked using the NanoPhotometer ${ }^{\circ}$ spectrophotometer (IMPLEN, CA, USA), RNA concentration was measured using Qubit ${ }^{\circ}$ RNA Assay Kit in Qubit $^{\oplus}$ 2.0 Flurometer (Life Technologies, CA, USA), and RNA $\geq 1 \mu \mathrm{g}$ without pollution can be prepared for normal library. RNA integrity was assessed using the RNA Nano 6000 Assay Kit of the Bioanalyzer 2100 system (Agilent Technologies, CA, USA), and the standard of quality control is that RIN value $\geq 7$ and $28 \mathrm{~S} / 18 \mathrm{~S}>0.7$.

\section{DGE library build}

A total amount of $3 \mu \mathrm{g}$ RNA per sample was used as input material for the RNA sample preparations. The mRNA was purified from total RNA using poly-T oligo-attached magnetic beads. After purification, the mRNA was randomly disrupted with a divalent cation in the NEB Fragmentation Buffer. The cDNA library was constructed by reverse transcribing the RNA fragment according to the NEB library (Figure 1). After the library was completed, initial quantification was performed using the Qubit 2.0 Fluorometer, and then the library was diluted to $1.5 \mathrm{ng} / \mu \mathrm{L}$. The insert size of the library was detected using the Agilent 2100 bioanalyzer. If the insert size was in accordance with expected concentration, precise concentration will be detected using qRT-PCR (effective concentration must be higher than $2 \mathrm{nM}$ ). After the quality control, detect the sample (Novegene company, China).

\section{Quality control and mapping}

Convert the original image file data to Sequenced Reads and then store it in FASTQ format. Calculate Q20, Q30 values and the error rate of each sample. At the same time, the GC content of the sample is calculated and removes reads containing adapter, reads containing ploy-N and low quality reads from raw data. Finally comparatively analyze the RNA-seq sequencing data using STAR software and map the clean reads to the transcript group.

\section{Quantitative analysis of gene expression}

HTSeq v0.6.0 was used to count the reads numbers mapped to each gene. And then FPKM of each gene was calculated based on the length of the gene and reads count mapped to this gene. FPKM, expected number of Fragments Per Kilobase of transcript sequence per Millions base pairs sequenced, considers the effect of sequencing depth and gene length for the reads count at the same time, and is currently the most commonly used method for estimating gene expression levels.

\section{Differential gene expression analysis}

Differential expression analysis of two groups was performed using the DESeq2 R package. DESeq2 provide statistical routines for determining differential expression in digital gene expression data using a model based on the negative binomial distribution. The resulting P-values were adjusted using the Benjamini and Hochberg's approach for controlling the false discovery rate. Genes with an adjusted P-value $<0.05$ found by DESeq 2 were assigned as differentially expressed.

\section{Enrichment analysis of differentially expressed genes}

Enrichment analysis of differentially expressed genes was implemented by the clusterProfiler R package. GO (Gene Ontology) is a database describing gene function, which can be divided into three parts: molecular function, biological process and cell composition. GO terms with corrected $P$ value less than 0.05 were considered significantly enriched by differential expressed genes. KEGG (Kyoto Encyclopedia of Genes and Genomes) is a database integrating genome, chemistry and system function information.

\section{Statistical Analysis}

SPSS 16.0 software (Chicago, USA) and Graphpad Prism 5 (San Diego, USA) was used for statistical data analysis. Qualitative data were presented as numbers and percentages. The normal distribution data were presented as mean \pm standard deviation. The non-normal distribution were presented as median (range). $\mathrm{P}$ values $<0.05$ were considered to be statistically significant, and $\mathrm{P}$ values $\geq 0.05$ were considered not significant (NS) (Figure 1).

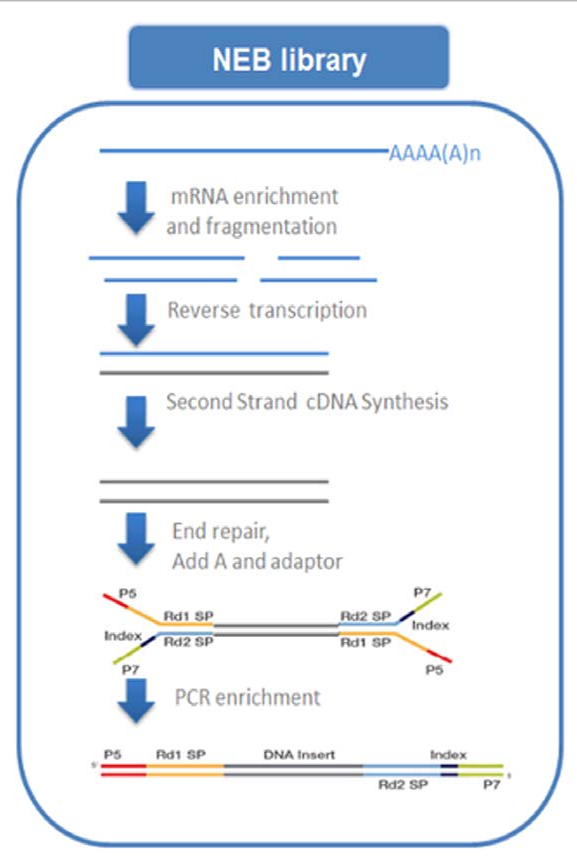

Figure 1: NEB general database. Sequencing Connector: Includes three parts: P5/P7, index and Rd1/Rd2 SP (as shown above). P5/P7 is the PCR primers and a part of flow cell combining with primer. The index provides $\mathrm{Rd} 1 / \mathrm{Rd} 2$ which can differentiate between library information. SP is the read1/ read2 sequence primer, which is the binding part of sequencing primers. The sequencing process theoretically start backwards from $\mathrm{Rd} 1 / \mathrm{Rd} 2 \mathrm{SP}$. 
Citation: Zhang W, Zuo M, Shen H, Yin J, Wang W, et al. (2018) Gene Expression Profiles of Programmed Death-1 (+) Lymphocytes in Peripheral Blood Reveal their Significance in Tumor Immunity of T Cell Non-Hodgkin Lymphoma. J Blood Lymph 8: 223. doi:10.4172/2165-7831.1000223

Page 3 of 7

\section{Result}

\section{Clinical data}

The median age of 10 newly diagnosed T-NHL patients was 32.5 years (12 to 67 years), and the male: female ratio was 1:1. According to Ann Arbor staging, $60 \%$ of patients were stage III to IV, while stage I II patients accounted for $40 \%$. Based on the subtype of lymphoma, $40 \%$ patients were NK/T cell lymphoma, followed by peripheral T-cell lymphoma (non-specific type) which account for 30\%. Besides, $70 \%$ of patients suffer multiple organ involvement (Table 1). 10 cases of healthy control were at the median age of 36.5 years (22 to 50 years) with male: female ratio of $1: 1$.

\section{Results of gene expression profiling}

Quantitative analysis: According to the FPKM value of each sample, the correlation coefficient between the patients group and the health controls group was calculated and presented as a heat map (Figure 2). As we can see in the Figure 2, there was difference between T-NHL patients (E1-E10) and healthy controls (C1-C10). Besides, the heterogeneity of T-NHL patients group was significantly stronger than that of the control group, and patients E3 and E9 were obviously different from other patients. Figure 2 is the PCA cluster diagram of 20 subjects, which intuitively shows that patients and healthy controls can be roughly divided into two groups and the individuals of patients

\begin{tabular}{|c|c|c|c|c|c|c|}
\hline Number & Gender & Age & Subtype & Stage & B symptoms & Involved Organ \\
\hline E1 & Male & 22 & PTCL, NOS & IV & Yes & subcutaneous tissue, CNS \\
\hline E2 & Female & 67 & NKTL & IV & No & Skin, lymph node, thyroid, lung \\
\hline E3 & Male & 19 & ALCL & IV & Yes & lymph node, bone marrow \\
\hline E4 & Male & 27 & SPTCL & IV & Yes & Skin, subcutaneous tissue \\
\hline E5 & Male & 46 & PTCL, NOS & IV & No & skin \\
\hline E6 & Male & 55 & NKTL & II & No & Nerve, muscle, nasal cavity, lymph node \\
\hline E7 & Female & 22 & PTCL, NOS & II & Yes & Lymph node, gastrointestinal system \\
\hline E8 & Female & 38 & NKTL & II & Yes & nasal cavity \\
\hline E9 & Female & 41 & NKTL & IV & Yes & Breast, subcutaneous tissue, nasal cavity, bone marrow \\
\hline E10 & Female & 12 & MF & II & Yes & skin \\
\hline
\end{tabular}

Abbreviation: PTCL; NOS: peripheral T-cell lymphoma, not otherwise specified; NKTL: NK/T Cell Lymphoma; ALCL: Anaplastic Large Cell Lymphoma; SPTCL: Subcutaneous Panniculitis-like T-Cell Lymphoma; MF: Mycosis Fungoides; CNS: Central Nerve System.

Table 1: Clinical characteristics of newly diagnosed T-NHL patients.

PCA 3D Plot

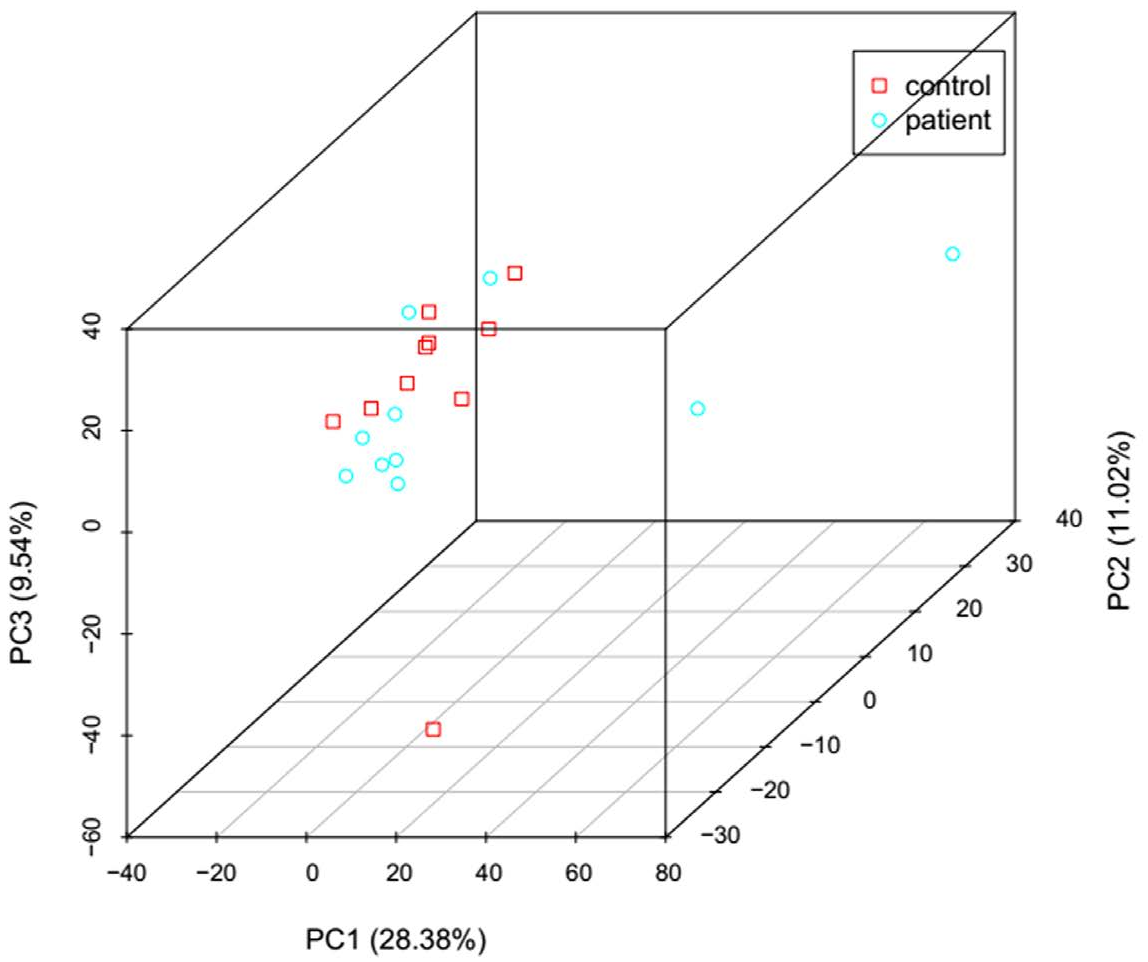

Figure 2: Principal component analysis (PCA) cluster diagram of sample. PCA utilize linear algebra to deal with tens thousands of genetic variables by dimensionality reduction and principal component extraction. PCA can also be used to assess differences between groups and reproducibility of sample within the group. Ideally, the samples within group should be clustered together and the samples between groups should be scattered in the PCA diagram. The red square represents healthy controls, and the blue circle represents T-NHL patients. 
Citation: Zhang W, Zuo M, Shen H, Yin J, Wang W, et al. (2018) Gene Expression Profiles of Programmed Death-1 (+) Lymphocytes in Peripheral Blood Reveal their Significance in Tumor Immunity of T Cell Non-Hodgkin Lymphoma. J Blood Lymph 8: 223. doi:10.4172/2165-7831.1000223

Page 4 of 7

group are more dispersed, among whom two patients were significantly different from others.

Differential gene expression analysis: Compared with the healthy control group, the number of differentially expressed genes in PD-1 (+) lymphocytes in T-NHL patients was 5195, among which 3321 genes were highly expressed and 1874 genes were low expressed. In order to better understand the difference in gene expression between the two groups of samples, we underwent the hierarchical clustering analysis of differential expressed gene (Figure 3). And the result showed that high expression and low expression gene region of two groups (T-NHL patients and healthy controls group) were significantly different, but gene expression of patient $\mathrm{E} 10$ was similar to that of healthy controls. In addition, gene expression pattern of T-NHL group was heterogeneous, according to which we could divide the patients into three groups: 1 ) E3 and E9; 2) E1, E4 and E5; 3) E2, E6, E7 and E8.

Enrichment analysis: 5915 differentially expressed genes were dealt with enrichment analysis, in order to further understand what kind of biological functions were correlated. GO enrichment analysis consist of three parts: molecular function, cell composition and biological process. After GO ID of genes were identified, the WEGO analysis was performed. The differentially expressed gene could be divided into 619 different functional groups. Figure 4 showed part of functional groups and the significance of difference. Actually most of these functional groups were belong to the biological process, involving the inherent immunity, T lymphocyte function and so on. In addition, KEGG enrichment analysis was performed to further understand the function and biological significance of differentially expressed genes. The results showed that these genes were associated with cell cycle, natural killer cell-mediated cytotoxicity, $\mathrm{T}$ cell receptor signaling pathway, MAPK signaling pathway and Toll-like Receptor signaling pathway, etc. (Figure 4).

\section{Discussion}

Programmed death factor-1 (PD-1) is an inhibitory immunostimulatory factor which can inhibits the proliferation and

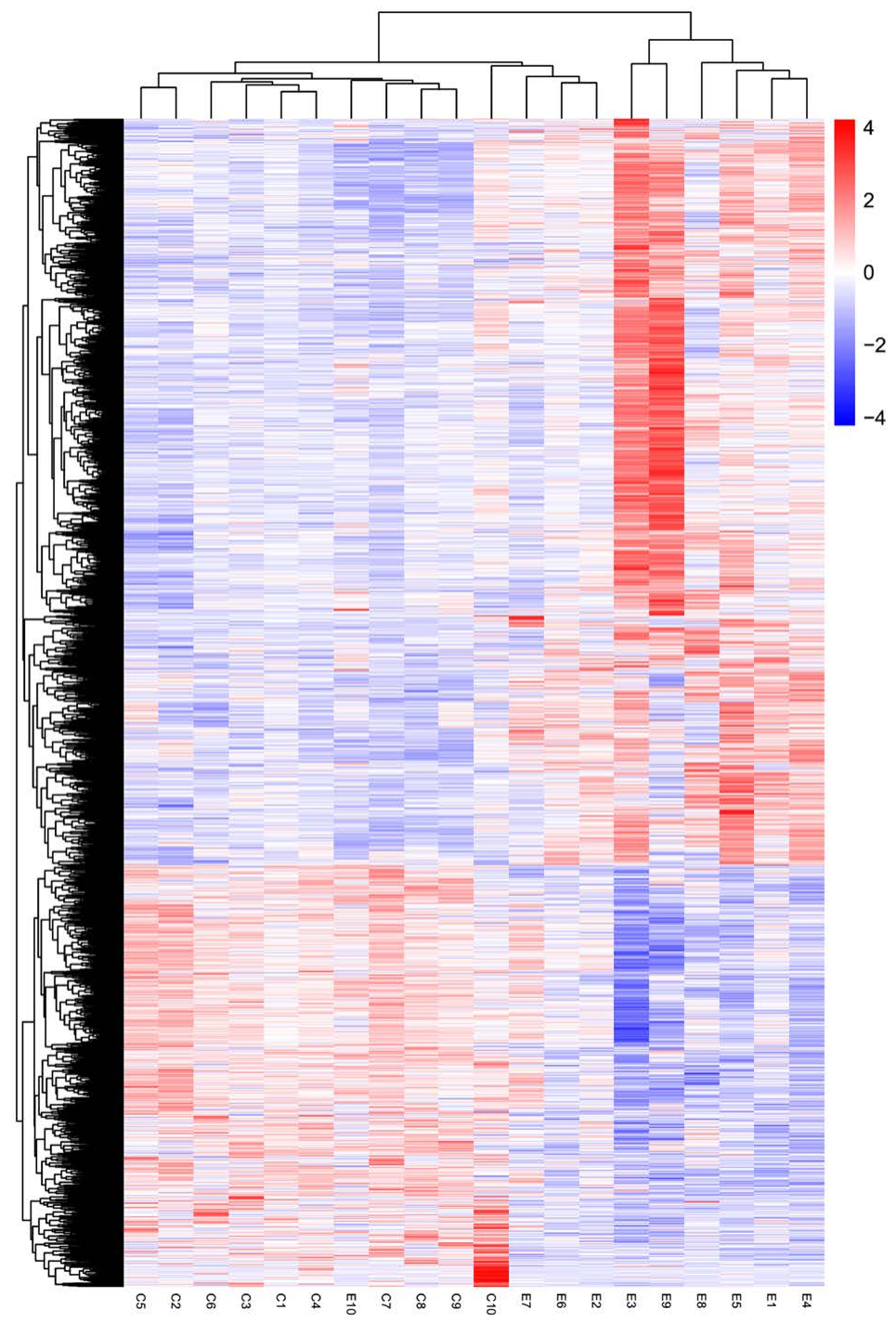

Figure 3: Hierarchical clustering heat map of differentially expressed gene. The abscissa indicates the sample number, the ordinate indicates different gene probe. The rectangular units indicate the sample gene expression level, which are normalized by log10 $(F P K M+1)$, and red indicate high expression, while blue indicate low expression. The right side of the graph shows the color scale and the corresponding log10 (FPKM +1$)$ value. 


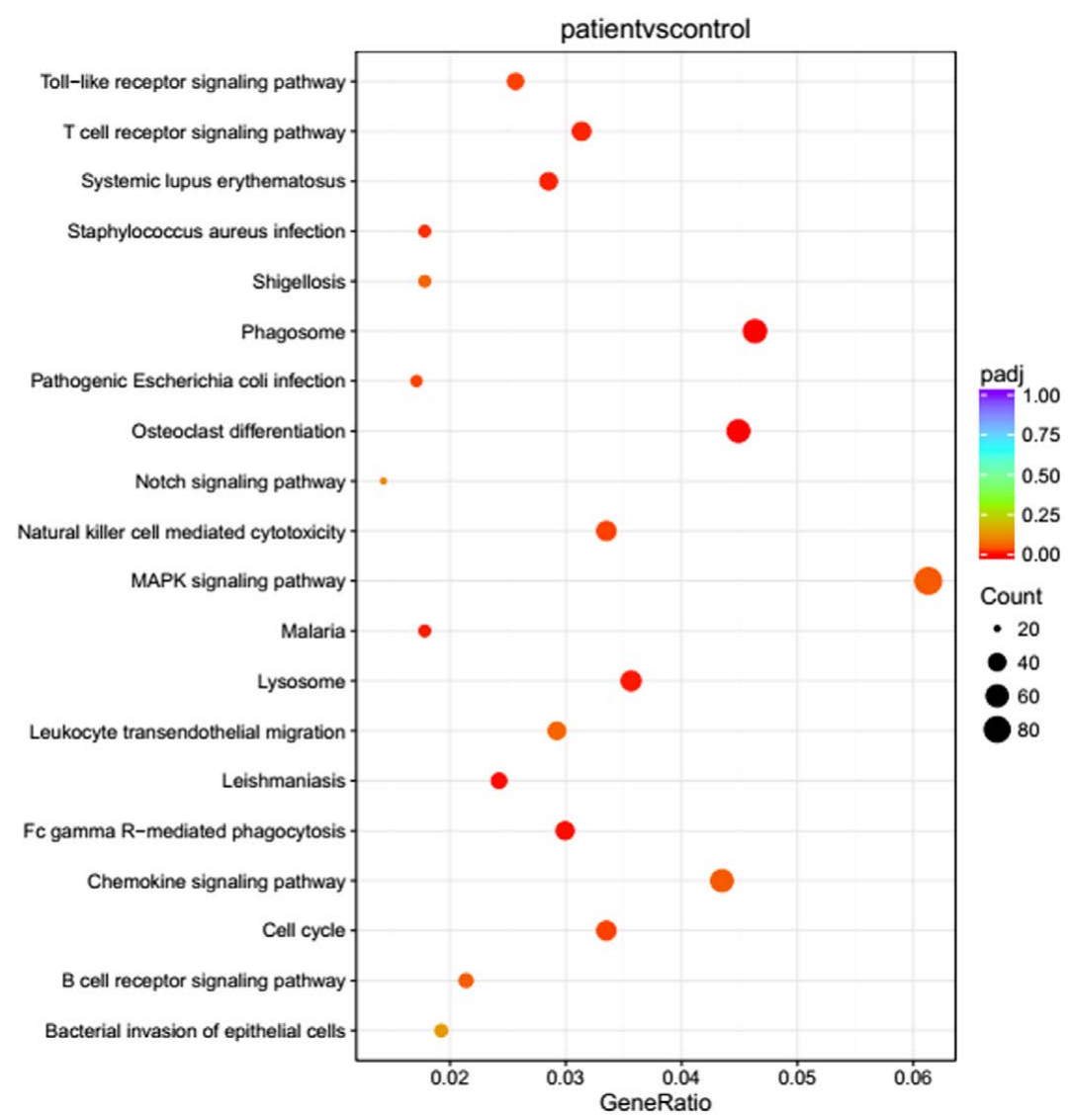

Figure 4: Enrichment analysis of differentially expressed genes. KEGG enrichment analysis scatter gram, the ordinate indicates the different biological functions, abscissa indicates the proportion of genes, and the larger the dot is, the greater the proportion of genes is. The right histogram indicates color gradation and the Padj value. Padj value $<0.05$ is regarded as significant difference.

function of varieties of immune cells. When PD-1 was stimulated, it could inhibits PI3K through downstream phosphatase SHP1/SHP2, finally inhibiting lymphocyte activation and proliferation related signal pathways [15]. In addition, it has been found that viral infection and interferon alpha (IFN- $\alpha$ ) can increase the expression of PD-1 on monocytes and macrophages, at the meantime affecting the release of cytokines in macrophages [16-18]. In the tumor microenvironment, the $\mathrm{PD}-1$ ligand, programed death ligand 1 (PD-L1), is expressed in tumor cells, and when it was combined with PD-1 on the lymphocytes, the function of immune cells were impaired, finally promoting the tumor immune escape, which is important for tumor research. But in T-NHL few studies have reported the significance of PD-1 expression in tumor immunity. Therefore, our study tried to figure out gene expression profile of peripheral blood PD-1 (+) lymphocytes, initially exploring the significance of circulating immune cells in tumor immunity.

The results showed that there were significant differences in PD-1 (+) lymphocytes between T-NHL patients and healthy controls. More than 5,000 genes were differentially expressed, and about $60 \%$ of genes were highly expressed in T-NHL patients, with $40 \%$ of genes lowly expressed, suggesting that PD-1 (+) lymphocytes in peripheral blood may play important roles in T-NHL patients. Besides, GO enrichment analysis showed that these differentially expressed genes may contribute to the development of tumor. The related biological processes included cell proliferation /apoptosis, immune response, signal transduction, substance metabolism and cell adhesion, and the majority of important differential functional groups are related to immune function, such as the activation of innate immune, regulation of immune response, regulation of $\mathrm{T}$ lymphocyte function and so on. Actually, the abnormal gene expression of innate immune cells (including DC cells, macrophages, etc.) and $\mathrm{T}$ lymphocytes may be associated with tumor and is relevant to antigen recognition and tumor immunoregulation. Studies [19-23] have confirmed that in the peripheral blood of lymphoma patients, the number and proportion of $\mathrm{T}$ lymphocytes (CD4, CD8 and Treg) and NK cells were significantly abnormal, and after the treatment gradually return to normal, supporting that just like the tumor microenvironment, peripheral blood immune cells may also participate the process of tumor immune escape.

In addition, KEEG enrichment analysis indicated that signaling pathways related to differential gene expression mainly include cell cycle, natural killer cell (NK cell) -mediated cytotoxicity, T cell receptor signaling pathway and Toll-like receptor signaling pathway. Dysregulated expression of Cyc, E2F, Rb, TGF $\beta$ and Smad in the cell cycle pathway result in abnormal synthesis of DNA and related protein, and finally affect the proliferation of immune cells. In the peripheral blood of T-NHL patients, the proliferative activity of inhibitory cells such as PD-1 (+) Treg cells may be enhanced, while the effector T cells are inhibited, so immune system is in inhibitory condition and is more compatible for tumorigenesis and development. In addition, the difference of $\mathrm{T}$-cell receptor signaling pathway and natural killer cell-mediated cytotoxicity between T-NHL patients from healthy 
Citation: Zhang W, Zuo M, Shen H, Yin J, Wang W, et al. (2018) Gene Expression Profiles of Programmed Death-1 (+) Lymphocytes in Peripheral Blood Reveal their Significance in Tumor Immunity of T Cell Non-Hodgkin Lymphoma. J Blood Lymph 8: 223. doi:10.4172/2165-7831.1000223

Page 6 of 7

individuals confirmed again that the peripheral immune status in T-NHL patients was significantly dysregulated.

Toll-like receptor signaling pathways are also important in the pathogenesis and progression of lymphoma. Eleonora Fonte [24] found that in vitro experiment the expression pattern of Toll-like receptor protein is correlated with activation, proliferation and proliferation of lymphoma cells in splenic marginal zone lymphoma, the activation of TLR1/2, TLR2/6 and TL9 can activate downstream NF- $\kappa B$, MAPK and IRAK kinase signaling pathway, promoting the development of lymphoma. At present, many studies attempt to use Toll-like receptor antagonists in the treatment of B-cell lymphoma, and have achieved good effect in animal experiments [25]. More than that, Jiang Zhu et al tried to combined toll-like receptor antagonist with doxorubicin in the treatment of $\mathrm{T}$ cell lymphoma mouse model, the results show that Toll-like receptor antagonist can significantly enhance the treatment of doxorubicin [26]. In this study, TLR1/2, TLR2/6, TLR4, TLR5 and its downstream MYD88, P38, MAPK signaling pathways were significantly activated in T-NHL patients, leading to activation of T cells and the abnormal chemotaxis of immune cells (DC cells, neutrophils and NK cells), which is consistent with the results of GO enrichment analysis. So it is implied that peripheral PD-1 (+) lymphocytes may participate in the process of tumor antigen recognition and immunization.

In addition, in T-NHL patients group gene expression of E3 and E9 was significantly different from other patients, and the reason may be that E3 and E9 patients had bone marrow involvement, so the proportion and function peripheral blood immune cell may be more likely to be abnormal. Besides, as for peripheral T-cell lymphoma (not specifically), gene expression pattern of E5 and E1 patients were different from that of E7 patients, suggesting that gene expression profile of peripheral PD-1 (+) lymphocyte may contribute to further classification of T-NHL in a biological way and understanding the differences in pathogenesis and progression between patients, which could help us not only understand more deeply what the nature of the disease is, but also improving individualized clinical treatment and prognosis of patients.

In conclusion, gene expression profile of PD-1 (+) lymphocyte in peripheral blood of T-NHL patients help us find out the research value of peripheral immune cells in tumor immunity of T-NHL. However, our study was still limited by the fact that the sample size is too small and critical mRNA level and protein expression are not detected and confirmed. In addition, as mentioned earlier, the description of the immune system can just provide a new direction for the study of T-NHL, only when we find out the causal relationship between peripheral immune cells and tumor can we bring breakthrough of disease diagnosis and therapy, which still need more studies.

\section{Acknowledgments}

This work was supplied by grant from CAMS Innovation Fund for Medical Sciences (CIFMS2016-12M-1-001), Capital Foundation of Medical Developments (CFMD016-2-4016) and Pumch Science Fund for Junior Faculty (2016-1.19). The authors would like to thank all the physicians from the Department of Hematology at PUMC hospital for kindly providing clinical information, and Xuan Wang \& HaO Cai for providing technical assistance.

\section{Novelty and Impact Statements}

We utilized DGE to elucidate the gene expression difference of peripheral blood PD-1 (+) lymphocyte between T-NHL patients and the healthy, and showed that PD-1 (+) lymphocytes might be closely related to tumor immunity. Compared with tumor microenvironment, peripheral blood might be more stable and detectable, so our finding actually provided a new sight for the study of tumor immunity and also may contributed to the therapy of T-NHL.

\section{Conflicts of Interest}

No potential conflicts of interest were disclosed. All authors have approved the manuscript for submission. We confirmed that the content of the manuscript has not been published, or submitted for publication elsewhere.

\section{References}

1. Vose J, Armitage J, Weisenburger D (2008) International peripheral T-cell and natural killer/T-cell lymphoma study: pathology findings and clinical outcomes. $\mathrm{J}$ Clin Oncol 26: 4124-4130.

2. Savage KJ, Chhanabhai M, Gascoyne RD, Connors JM (2004) Characterization of peripheral T-cell lymphomas in a single North American institution by the WHO classification. Annals of Oncology 15: 1467-1475.

3. Abouyabis AN, Shenoy PJ, Lechowicz MJ, Flowers CR (2008) Incidence and outcomes of the peripheral T-cell lymphoma subtypes in the United States. Leuk Lymphoma 49: 2099-2107.

4. Kerkar SP, Restifo NP (2012) Cellular constituents of immune escape within the tumor microenvironment. Cancer Res 72: 3125-3130.

5. Ishida Y, Agata Y, Shibahara K, Honjo T (1992) Induced expression of PD-1, a novel member of the immunoglobulin gene superfamily, upon programmed cell death. EMBO J 11: 3887-3895.

6. Keir ME, Butte MJ, Freeman GJ, Sharpe AH (2008) PD-1 and its ligands in tolerance and immunity. Annu Rev Immunol 26: 677-704.

7. Taube JM, Klein A, Brahmer JR, Xu H, Pan X, et al. (2014) Association of PD-1, PD-1 ligands, and other features of the tumor immune microenvironment with response to anti-PD-1 therapy. Clin Cancer Res 20: 5064-5074.

8. Lesokhin AM, Ansell SM, Armand P, Scott EC, Halwani A, et al. (2014) Preliminary Results of a Phase I Study of Nivolumab (BMS-936558) in Patients with Relapsed or Refractory Lymphoid Malignancies. Blood 124: 291.

9. Armand P, Nagler A, Weller EA, Devine SM, Avigan DE, et al. (2013) Disabling immune tolerance by programmed death-1 blockade with pidilizumab after autologous hematopoietic stem-cell transplantation for diffuse large B-cel lymphoma: results of an international phase II trial. J Clin Oncol 31: 4199-4206.

10. Berger R, Rotem-Yehudar R, Slama G, Landes S, Kneller A, et al. (2008) Phase I safety and pharmacokinetic study of CT-011, a humanized antibody interacting with $\mathrm{PD}-1$, in patients with advanced hematologic malignancies. Clin Cancer Res 14: 3044-3051.

11. Zhang $L$ (2015) Prognostic value of PD-1 and TIM-3 on CD3+ T cells from diffuse large B-cell lymphoma. Biomed Pharmacother 75: 83-87.

12. Oki Y, Buglio D, Zhang J, Ying Y, Zhou S, et al. (2014) Immune regulatory effects of panobinostat in patients with Hodgkin lymphoma through modulation of serum cytokine levels and T-cell PD1 expression. Blood Cancer J 4: e236.

13. Ni L, Ma CJ, Zhang Y, Nandakumar S, Zhang CL, et al. (2011) PD-1 modulates regulatory $\mathrm{T}$ cells and suppresses $\mathrm{T}$-cell responses in $\mathrm{HCV}$-associated lymphoma. Immunol Cell Biol 89: 535-539.

14. Swerdlow SH, Campo E, Harris NL, Jaffe ES, Pileri SA, et al. (2008) WHO Classification of Tumours of Haematopoietic and Lymphoid Tissues, Fourth Edition.

15. Nishimura H, Okazaki T, Tanaka Y, Nakatani K, Hara M, et al. (2001) Autoimmune dilated cardiomyopathy in $\mathrm{PD}-1$ receptor-deficient mice. Science 291: 319-322.

16. Said EA, Dupuy FP, Trautmann L, Zhang Y, Shi Y, et al. (2010) Programmed death-1-induced interleukin-10 production by monocytes impairs CD4+ $T$ cell activation during HIV infection. Nat Med 16: 452-459.

17. Cho H, Choi EK, Lee SW, Jung KO, Seo SK, et al. (2009) Programmed death-1 receptor negatively regulates LPS-mediated IL-12 production and differentiation of murine macrophage RAW264.7 cells. Immunol Lett 127: 39-47.

18. Cho H, Lee SW, Seo SK, Choi IW, Choi I, et al. (2008) Interferon-sensitive response element (ISRE) is mainly responsible for IFN-alpha-induced upregulation of programmed death-1 (PD-1) in macrophages. Biochim Biophys Acta 1779: 811-819.

19. Zhang X, Xu J, Zhu HY, Wang Y, Wang L, et al. (2016) Negative prognostic impact of low absolute CD4+ T cell counts in peripheral blood in mantle cell lymphoma. Cancer Sci 107: 1471-1476.

20. Gunduz E, Sermet S, Musmul A (2016) Peripheral blood regulatory T cell levels are correlated with some poor prognostic markers in newly diagnosed lymphoma patients. Clinical cytometry 90: 449-454. 
Citation: Zhang W, Zuo M, Shen H, Yin J, Wang W, et al. (2018) Gene Expression Profiles of Programmed Death-1 (+) Lymphocytes in Peripheral Blood Reveal their Significance in Tumor Immunity of T Cell Non-Hodgkin Lymphoma. J Blood Lymph 8: 223. doi:10.4172/2165-7831.1000223

Page 7 of 7

21. Lu T, Yu S, Liu Y, Yin C, Ye J, et al. (2016) Aberrant Circulating Th17 Cells in Patients with B-Cell Non-Hodgkin's Lymphoma. Plos one 11: e0148044.

22. Yin $Q$, Chen L, Li Q, Mi R, Li Y, et al. (2014) Changes of T-lymphocyte subpopulation and differential expression pattern of the T-bet and GATA-3 genes in diffuse large B-cell lymphoma patients after chemotherapy. Cancer Cell Int 14: 85.

23. Shafer D, Smith MR, Borghaei H, Millenson MM, Tianyu Li, et al. (2013) Low NK cell counts in peripheral blood are associated with inferior overall survival in patients with follicular lymphoma. Leukemia research 37: 1213-1215.
24. Fonte E, Agathangelidis A, Reverberi D, Ntoufa S, Scarfò L, et al. (2015) Tolllike receptor stimulation in splenic marginal zone lymphoma can modulate cell signaling, activation and proliferation. Haematologica 100: 1460-1468.

25. Arunkumar N, Liu C, Hang H, Song W (2013) Toll-like receptor agonists induce apoptosis in mouse B-cell lymphoma cells by altering NF-kappaB activation. Cell Mol Immunol 10: 360-372.

26. Zhu J, He S, Du J, Wang Z, Li W, et al. (2015) Local administration of a nove Toll-like receptor 7 agonist in combination with doxorubicin induces durable tumouricidal effects in a murine model of T cell lymphoma. J Hematol Oncol 8: 21. 